\title{
Diversification of the molecular clockwork for tissue-specific function: insight from a novel Drosophila Clock mutant homologous to a mouse Clock allele
}

\author{
Eunjoo Cho ${ }^{1,2}$, Euna Lee ${ }^{1,3} \mathcal{E}$ Eun Young Kim ${ }^{1,2,3, *}$ \\ ${ }^{1}$ Department of Brain Science, ${ }^{2}$ Chronic Inflammatory Disease Research Center, ${ }^{3}$ BK21 Plus program, Neuroscience Graduate Program, \\ Department of Biomedical Sciences, Ajou University School of Medicine, Suwon 16499, Korea
}

\begin{abstract}
The circadian clock system enables organisms to anticipate the rhythmic environmental changes and to manifest behavior and physiology at advantageous times of the day. Transcriptional/ translational feedback loop (TTFL) is the basic feature of the eukaryotic circadian clock and is based on the rhythmic association of circadian transcriptional activator and repressor. In Drosophila, repression of dCLOCK/CYCLE (dCLK/CYC) mediated transcription by PERIOD (PER) is critical for inducing circadian rhythms of gene expression. Pacemaker neurons in the brain control specific circadian behaviors upon environmental timing cues such as light and temperature cycle. We show that amino acids 657-707 of dCLK are important for the transcriptional activation and the association with PER both in vitro and in vivo. Flies expressing dCLK lacking AA657-707 in $\mathrm{Clk}^{\text {out }}$ genetic background, homologous to the mouse Clock allele where exon 19 region is deleted, display pacemaker-neuron-dependent perturbation of the molecular clockwork. The molecular rhythms in light-cycle-sensitive pacemaker neurons such as ventral lateral neurons $\left(L N_{v} s\right)$ were significantly disrupted, but those in temperature-cycle-sensitive pacemaker neurons such as dorsal neurons (DNs) were robust. Our results suggest that the dCLK-controlled TTFL diversify in a pacemaker-neuron-dependent manner which may contribute to specific functions such as different sensitivities to entraining cues. [BMB Reports 2016; 49(11): 587-589]
\end{abstract}

*Corresponding author. E-mail: ekim@ajou.ac.kr

https://doi.org/10.5483/BMBRep.2016.49.11.178

Received 17 October 2016

Keywords: Circadian rhythm, CLOCK, Drosophila, PERIOD, TTFL

Abbreviations: $\mathrm{DN}$, dorsal neuron; $\mathrm{LN}_{\mathrm{V}}$, ventral lateral neuron; SCN, suprachiasmatic nucleus; TTFL, Transcriptional/Translational Feedback Loop

Perspective to: Euna Lee, 2016, Pacemaker-neuron-dependent disturbance of the molecular clockwork by a Drosophila CLOCK mutant homologous to the mouse Clock mutation. Proc Natl Acad Sci U S A 113(33):E4904-13, 10.1073/pnas.1523494113

\section{INTRODUCTION}

Organisms are able to harmonize their physiology and behavior with cyclic environmental changes by means of circadian timing system composed of cell-autonomous molecular oscillators generated by transcriptional/translational feedback loops (TTFLs). Numerous genetic and biochemical studies performed in model organisms including fungi, fruit flies, and mice have identified core components and mechanistic underpinnings of circadian oscillators. While molecular components of circadian oscillator vary among organisms, the fundamental basis of the self-sustained cellular circadian oscillator, the negative feedback between transcriptional activators and repressors is well conserved. CLOCK is a rate-limiting component of the TTFL in both fruit flies and mammals. In flies, dCLOCK (Drosophila CLOCK, dCLK) and CYCLE (CYC) form a heterodimer to activate circadian gene expression of core clock genes including transcription repressors period (per) and timeless (tim) and downstream clock controlled genes (ccgs). PER and TIM heterodimers translocate to the nucleus and physically interact with $\mathrm{dCLK} / \mathrm{CYC}$ to inhibit dCLK/CYC-dependent transcription.

To further understand the transcriptional repression of dCLK by PER, we sought to identify the region required for PER interaction using series of $\mathrm{dCLK}$ internal deletion mutants. We have identified the small region (AA657-707) in dCLK is required for the PER interaction. Interestingly, this domain turned out to be homologous to the region encoded by exon 19 of mouse Clock, which is deleted in Clock/Clock mutant mice. Thus, we have also confirmed that the region encoded by exon 19 of $\mathrm{mCLK}$ is required for the interaction with three mPER proteins (mPER1, mPER2, mPER3) in mammalian cells, suggesting the biochemical feature of interaction between PER and CLK is conserved in flies and mammals. It has been well known that mCLK internally deleted with the exon 19encoded region has reduced transcriptional activity (Gekakis et al., 1998, Science 280 (5369):1564-9, DOI: 10.1126/science. 280.5369.1564). Similar to mCLK, dCLK also requires AA657707 for its transcriptional activity since dCLK- $\Delta$, which lack AA656-707, did not induce expression of a luciferase reporter 
in S2 cells. Next, we sought to clarify whether AA657-707 of $\mathrm{dCLK}$ is sufficient for direct PER binding. While the AA657707 fragment tagged with GST failed to strongly associate with PER, GST-tagged AA380-528 fragment exhibited strong binding with PER indicating that AA657-707 of dCLK might not be sufficient for direct binding with PER. Nonetheless, we showed that AA657-707 of dCLK is clearly important for dCLK transcriptional activity and interacting with PER; moreover, this region is homologous to the exon 19 region of $\mathrm{mCLK}$.

To investigate the in vivo significance of AA657-707 of $\mathrm{dCLK}$, transgenic flies expressing dCLK- $\Delta$ was generated and introduced in $d C l k$ null $C l k^{\text {out }}$ genetic background (herein termed as $\left.\mathrm{p}\{d C l k-\Delta\} ; C l k^{\text {out }}\right)$. $\mathrm{p}\{d C l k-\Delta\} ; C l k^{\text {out }}$ flies showed no morning or evening anticipatory activities during the photic cycle and completely arrhythmic locomotor activities during constant dark condition. We then checked the biochemical attributes of $d C L K-\Delta$ in flies. Impaired binding of dCLK- $\Delta$ to PER was confirmed by immunoprecipitation assays using fly-head extracts. Consistent with the low transcriptional activity of dCLK- $\Delta$ in S2 cells, mRNA levels of dCLK target genes, such as per, tim, and vrille (vri), showed dampened oscillation than the case of control flies. However, oscillations of PER and TIM protein from fly heads were comparable to those of control flies in the photic cycle. We have reasoned that the discrepancy between quasi-normal protein oscillation of PER/TIM and the arrhythmic behavior in photic entrainment condition may come from pacemaker neuron-specific alterations of PER/TIM protein levels in $p\{d C l k-\Delta\} ; C l k^{\text {out }}$ flies.

In flies, about 150 anatomically distinct pacemaker neurons are located in the lateral and dorsal parts of the brain. Large ventral lateral neurons $\left(I L N_{v} s\right)$ respond to light and play a crucial role mediating photic entrainment. After entrainment, rhythms of locomotor activities are controlled by morning oscillators composed of PDF-positive small $\mathrm{LN}_{\mathrm{v}} \mathrm{s}$ and evening oscillators composed of $\mathrm{LN}_{\mathrm{d} S}, 5^{\text {th }} \mathrm{LN}_{\mathrm{v}}$, and some dorsal neurons $\left(\mathrm{DN} \mathrm{N}_{1} \mathrm{~s}\right)$. On the other hand, dorsal neurons $(\mathrm{DNs})$ are known to play a prominent role in entrainment to temperature cycles which serve as effective entrainment cue to synchronize circadian rhythms as well. We have examined the molecular oscillation of PER and TIM in each of these clusters of pacemaker neurons. Surprisingly, while control flies show synchronized strong amplitude of molecular oscillation in all pacemaker neurons, $\mathrm{p}\{d C l k-\Delta\} ; C l k^{\text {out }}$ flies showed different alterations of molecular rhythms depending on pacemaker neurons. Both PER and TIM showed robust oscillation in DNs. The amplitude of oscillations in the levels of PER and TIM was greatly reduced in $L_{v} s$. Since $L N_{v} s$ are important for photic entrainment, dramatically dampened the oscillation of PER and TIM proteins in $L_{\mathrm{v}} \mathrm{S}$ accounts for the behavioral arrhythmicity in photic entrainment condition. Quasi-normal oscillation of CLK target proteins in DNs of $p\{d C l k-\Delta\} ; C l k^{\text {out }}$ flies led us to hypothesize that these flies could be well entrained to temperature cycles. Indeed, $\mathrm{p}\{d C l k-\Delta\} ; C l k^{\text {out }}$ flies exhibited rhythmic locomotor behaviors anticipating warm and cold temperature transitions yet with slightly delayed cryo-phase activities. Consistent with delayed rhythmic locomotor behaviors, molecular rhythms of PER and TIM proteins in DNs showed robust oscillation with a phase delay.

Intriguingly, this $\mathrm{p}\{d C l k-\Delta\} ; C l k^{\text {out }}$ flies locomotor behaviors show a sharp contrast with those of $p\{d C l k-15 \mathrm{~A}\} ; C l k^{\text {out }}$ flies where all the mapped phospho-sites were switched to alanine (dCLK-15A). p $\{d C l k-15 \mathrm{~A}\} ; C l k^{\text {out }}$ flies are normally synchronized to photic entrainment but not to temperature entrainment (Lee et al., 2014 PLoS Genet 10: e1004545, DOI. 10.1371/journal. pgen.1004545). Considering highly conserved molecular mechanisms of core clock machinery, differential perturbations depending on pacemaker neurons by different mutations of $\mathrm{dCLK}$ protein are very surprising and unexpected. However, studies have accumulated to show that core circadian transcriptional complexes have different forms in individual cell-types to execute tissue-specific functions. For example, real-time reporting of PER2::LUC in culture isolated from individual tissues such as SCN, liver, lung, etc. manifested unique circadian period and phase suggesting that quantitative and/or qualitative characteristics of cellular oscillators might be different in a tissue-specific manner (Yoo et al., 2004 Proc Natl Acad Sci U S A. 101(15):5339-46, doi: 10.1073/pnas. 0308709101).

Questions remain regarding how $\mathrm{dCLK}-\Delta$ function differently

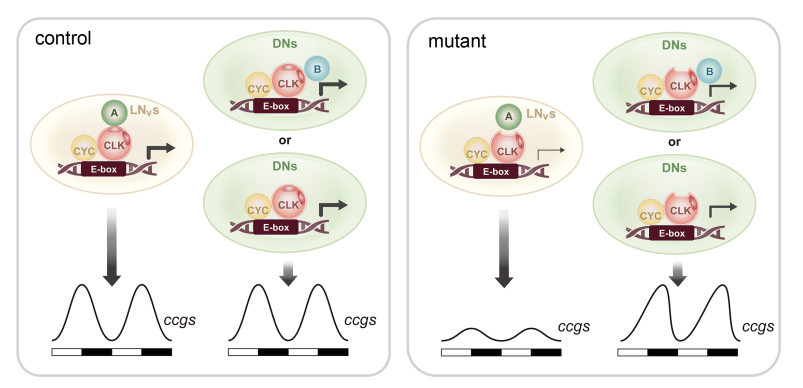

Fig. 1. Schematic model for tissue-specific molecular clockwork in $\mathrm{LN}_{\mathrm{v}} \mathrm{S}$ vs. DNs in Drosophila. In control flies, $\mathrm{LN}_{\mathrm{v}} \mathrm{s}$ specific coactivator A (green circle) binds to the region of dCLK spanning amino acids 657-707 (dashed area) and cooperate the dCLK-CYC dependent E-box-mediated transcription. On the other hand in $\mathrm{DNs}$, either coactivator B (blue circle) binds to the other part of dCLK (dotted area) cooperating the dCLK-CYC dependent E-boxmediated transcription or no co-activator is required. Therefore, in mutant flies, dCLK- $\Delta$ deficient in association with $L_{v}$ specific co-activator cannot support the strong amplitude of clock controlled genes (ccgs) expression. However, in DNs dCLK- $\Delta$ still induces transcription of ccgs yet with slightly delayed peak phase of expression. Please note that although we do not add schematic diagram here, an alternative model is possible. The intrinsic quantitative properties of $\mathrm{LN}_{\mathrm{v}}$ and DN TTFL might be different. So the vulnerability of $\mathrm{LN}_{\mathrm{v}}$ and $\mathrm{DN}$ oscillator to the disruption by reduced CLK transcriptional activity and/or CLK binding affinity to PER might be different resulting in totally different outcomes of circadian transcription. 
in $\mathrm{LN}_{\mathrm{v}} \mathrm{s}$ vs. DNs. We can think of two possibilities. First, as simplified in Fig. 1, there might be $\mathrm{LN}_{\mathrm{v}}$ or $\mathrm{DN}$-specific coactivators required for the dCLK dependent transcriptional activation. Deletion of AA657-707 may abolish the cell-type specific associations with specific coactivators leading to inefficient upregulation of clock-controlled genes in $\mathrm{LN}_{\mathrm{v}} \mathrm{s}$ but not in DNs. Second, due to the intrinsic properties of clockwork in individual tissues, the extent of reduction in transcriptional activity and/or repressor binding caused by $d C l k-\Delta$ mutation may be tolerable for some cell types (e.g. DNs in our study) but not so in other cells (e.g. $\mathrm{LN}_{\mathrm{v}} \mathrm{s}$ in our study). Obviously, further studies are needed to sort out these possibilities to provide a complete understanding of clockwork diversification which might evolve for tissue-specific function.

\section{ACKNOWLEDGEMENTS}

E.Y. Kim was supported by the National Research Foundation of Korea (NRF) grant (NRF-2014R1A2A1A11051765) and Chronic Inflammatory Disease Research Center (NRF-2012R1A5A0481 83) funded by the Korean government. E. Cho was supported by NRF grant (NRF-2013R1A1A2060533) funded by the Korean government. E. Lee and E.Y. Kim were supported by BK21 Plus Program from Ministry of Education, Korea. 